\title{
EVALUATION OF SOME SOILS IN NORTH WESTERN PARIS OASIS (EGYPT) USING STORIE INDEX AND SYS MODELS
}

\author{
Y. K. EL GHONAMEY \\ RS and GIS Unit, Soils, Water and Environment Research Institute -ARC - Giza
}

(Manuscript received 18 September 2014)

\begin{abstract}
$\mathrm{T}$ he North Western of Paris Oases represents one of the highest priority areas for future development in the country. The study area is located between longitudes $30^{\circ}$ $11^{\prime} 34.9^{\prime \prime}$ to $30^{\circ} 26^{\prime} 59.4^{\prime \prime}$ East and latitudes $24^{\circ} 40^{\prime} 9.3^{\prime \prime}$ to $24^{\circ} 51^{\prime}$ 8.7" North and covers an area about 123966 feddans. The purpose of this study is to evaluate some soils in north western Paris oasis using Remote Sensing (RS) and Geographic Information System (GIS). For this purpose, Forty-Three soil profiles were described in the field and their representative samples were analyzed. Using geomorpholical map, geological map and visual interpretation of satellite data a physiographic soil map was created to present mapping units of the study area. The area under investigation was classified into three landscape units, i.e. Plain, Dunes and Hills. Soil characteristics of the obtained mapping units were discussed and soil taxonomic unit were identified. Two models of land capability were used to evaluate the soils of study area. According to Storie Index model, the area under investigation was classified into three capability grades reflect the limitation factors, i.e. grade 1 (67.4 $\%)$, grade $3(26.16 \%)$ and grade $6(6.44 \%)$. on the other hand and according to Sys model the study area was classified into three capability classes, i.e. $S_{2}, S_{3}$ and $N_{2}$. The soils of $S_{2}$ have moderate limitations for agricultural crops, where texture is the main limiting factor ( $67.4 \%$ of the total area). The main limiting factors of soils of $\mathrm{S}_{3}$ are texture, depth and salinity $(26.16 \%)$, while the soils of $\mathrm{N}_{2}$ (6.44 \% of the total study area) include sand dunes, rock crops and shallow to very shallow soils. Five crops were selected to assess soil suitability for cultivation in the study area, i.e. wheat, barley, maize, tomato and olive. The results indicated that olive was more suitable for growing in such soils.
\end{abstract}

\section{INTRODUCTION}

The rapidly growing population in Egypt has a negative impact on its limited natural resources, including water and cultivated area. This requires proper management of such resources. The agricultural expansion outside the Nile Valley is one of the main objects of the Egyptian national plan (Darwish et. al., 2006).

One of the ways to meet population needs is to face this negative impact by increasing production per unit area and to utilize the land with respect to its potentiality in an appropriate way. Any utilization of the land over its capability will cause soil degradation and yield reduction . 
Remote sensing is defined as the acquisition of information about an object without being in physical contact with it (Elachi and Zyl, 2006). Therefore, the intrinsic characteristics of agriculture make remote sensing an ideal technique for its monitoring and management (Zhongxin et. al., 2004). Geographic Information System (GIS) is considered as organized collection of computer hardware, software and spatial and non-spatial data that can help users for the efficient capture, storage, update, manipulation, analysis and management of all geographically referenced information. Remote Sensing in combination with GIS techniques proved to be effective in sustainability and planning studies (DeVries, 1985).

The fundamental principle of land evaluation is to estimate the potential of a land for different productive uses, such as farming, livestock production, or forestry, together with uses that provide services or other benefits, such as water catchment, recreation, tourism and wildlife conservation (Dent and Young, 1981). Consequently, land evaluation is a tool for strategic land use planning. A specific agricultural use and management system on land that is most suitable according to agro-ecological potentialities and limitations is the best way to achieve sustainability (FAO, 1976 b).

Land capability is very important step in the reclamation process of the desert to determine the capability of soil cultivation to meet the requirement of the population. To make the evaluation two models were used, the first is Storie Index (Storie, 1978) which revised by O'Geen and Southard (2005), and the second is sys rating systems a methodology produced by Sys et. al. (1991).

The Storie Index express numerically the relative degree of suitability of a soil for agricultural uses. The Storie Index assesses the productivity of a soil based on soil characteristics obtained by evaluating soil surface, depth of the soil, texture of the surface layer, slope, and manageable factors (drainage and salts). Also, the Sys rating systems were suggested under the structure of the FAO Framework for Land Evaluation (FAO, 1976 b). Moussa (1991) indicated that the Storie index and Sys system could be considered as favorable systems under the conditions prevailing in the soil of Egypt.

This present study aims to evaluate land resources of the study area as well as producing land capability map for irrigated agriculture and land suitability map for specific crops. 


\section{MATERIALS AND METHODS}

\section{General description of the study area}

a) Location:

The study area is located in the south western desert in north west Paris oasis (Figure 1) between longitudes $30^{\circ} 11^{\prime} 34.9^{\prime \prime}$ to $30^{\circ} 26^{\prime} 59.4^{\prime \prime}$ East and latitudes $24^{\circ} 40^{\prime} 9.3^{\prime \prime}$ to $24^{\circ} 51^{\prime} 8.7^{\prime \prime}$ North and covers an area of about 123966 feddans.

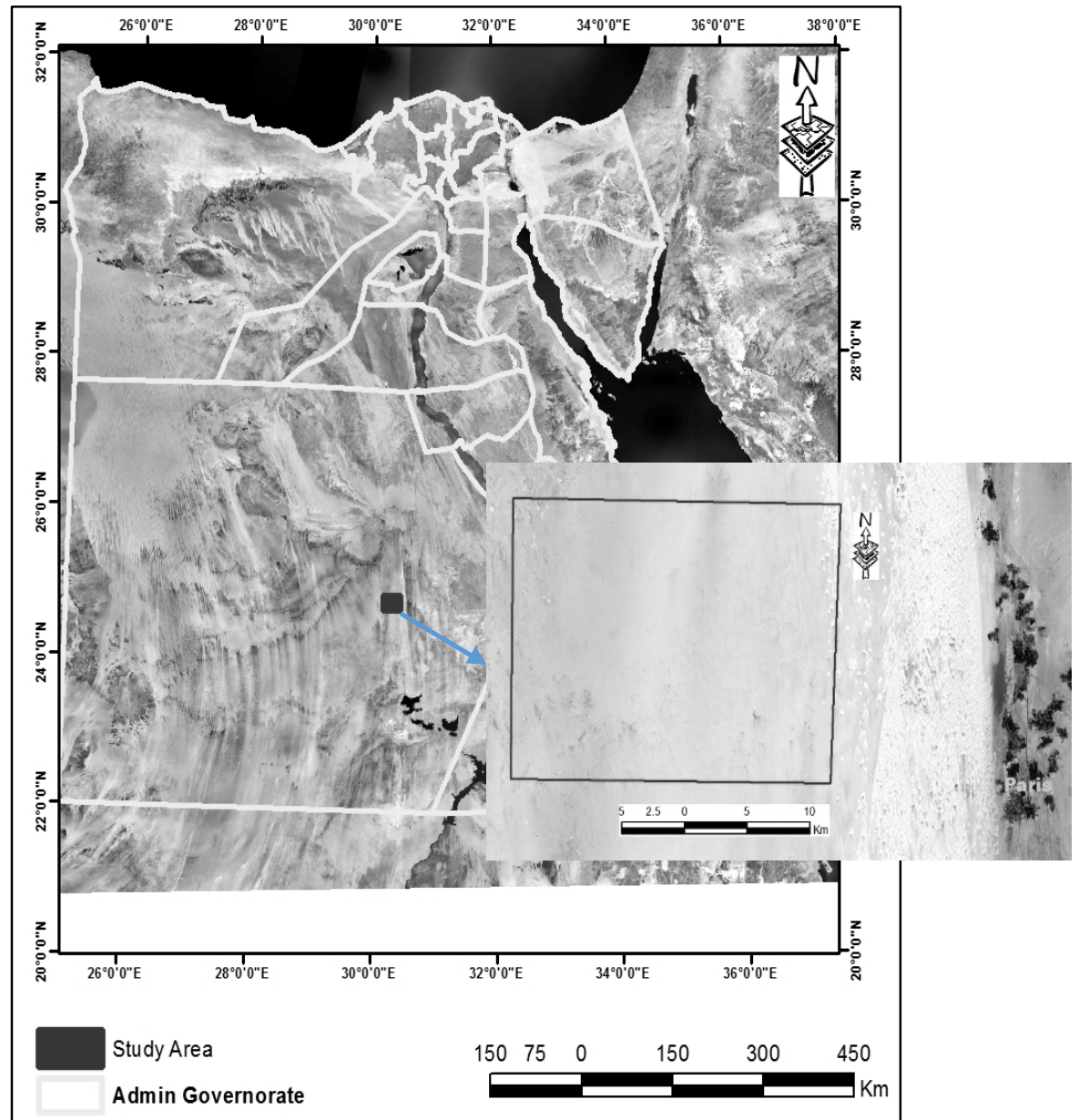

Figure 1. Location map of the study area.

b) Climate:

The area is characterized by a hot and dry summer with rare winter rainfall and bright sunshine through the year. The average annual temperature is $26.4{ }^{\circ} \mathrm{C}$, while the average of evaporation is $7.76 \mathrm{~mm}$ (Table 1). 
Table 1. The climitological norms of the study area (El Kharaga oasis meteorological station).

\begin{tabular}{|c|c|c|c|c|c|c|c|}
\hline \multirow[t]{2}{*}{ Month } & \multicolumn{2}{|c|}{ Temperature ${ }^{\circ} \mathbf{C}$} & \multirow{2}{*}{$\begin{array}{c}\text { Relative } \\
\text { Humidity } \\
(\%)\end{array}$} & \multirow{2}{*}{$\begin{array}{l}\text { Wind } \\
\text { Speed } \\
(\mathrm{m} / \mathrm{hr})\end{array}$} & \multirow{2}{*}{$\begin{array}{l}\text { Sun } \\
\text { shine } \\
\text { (hr) }\end{array}$} & \multirow{2}{*}{$\begin{array}{l}\text { Rain } \\
(\mathrm{mm})\end{array}$} & \multirow{2}{*}{$\begin{array}{c}\text { Evaporation } \\
(\mathrm{mm})\end{array}$} \\
\hline & highest & lowest & & & & & \\
\hline January & 24.6 & 9.2 & 37 & 2.5 & 8.0 & 0.1 & 6.0 \\
\hline February & 27.7 & 10.6 & 27 & 2.7 & 8.5 & · & 5.4 \\
\hline March & 32.9 & 15 & 19 & 3.0 & 10.0 & · & 6.0 \\
\hline April & 35.9 & 18 & 17 & 3.1 & 10.4 & · & 5.4 \\
\hline May & 39.4 & 21.9 & 15 & 3.0 & 10.9 & · & 8.0 \\
\hline June & 42.4 & 24.5 & 14 & 2.4 & 12.6 & · & 10.4 \\
\hline July & 42.3 & 24.6 & 16 & 2.3 & 12.1 & · & 8.7 \\
\hline August & 44.1 & 25.3 & 17 & 2.7 & 10.1 & · & 9.1 \\
\hline September & 40.3 & 23.7 & 20 & 2.5 & 8.7 & · & 9.9 \\
\hline October & 34.6 & 19.4 & 23 & 2.6 & 8.4 & · & 9.9 \\
\hline November & 29.5 & 14.3 & 36 & 2.5 & 8.1 & · & 7.7 \\
\hline December & 24.3 & 9.7 & 38 & 2.7 & 8.0 & 0.1 & 6.6 \\
\hline Average & 34.8 & 18.0 & 23.3 & 2.7 & 9.7 & 0.02 & 7.76 \\
\hline
\end{tabular}

* Meteorological Authority, 2014 .

c) Geology:

According to the geological map (scale 1: 500000), produced by EGSA (1988) the sand sheets serir is the dominant formation which represents an area of about 89158 feddans (71.92\%) of the total study area, covering the east part, followed by Sabaya Formation (Desert Rose Beds) representing an area of 32515 feddans (26.23 $\%)$ of the total study area, which concentrated in the western part while sand dunes while sabkha deposits cover small part in east of study area (2293 feddans represent $1.85 \%$ of the study area) (Figure 2 and Table 2). 


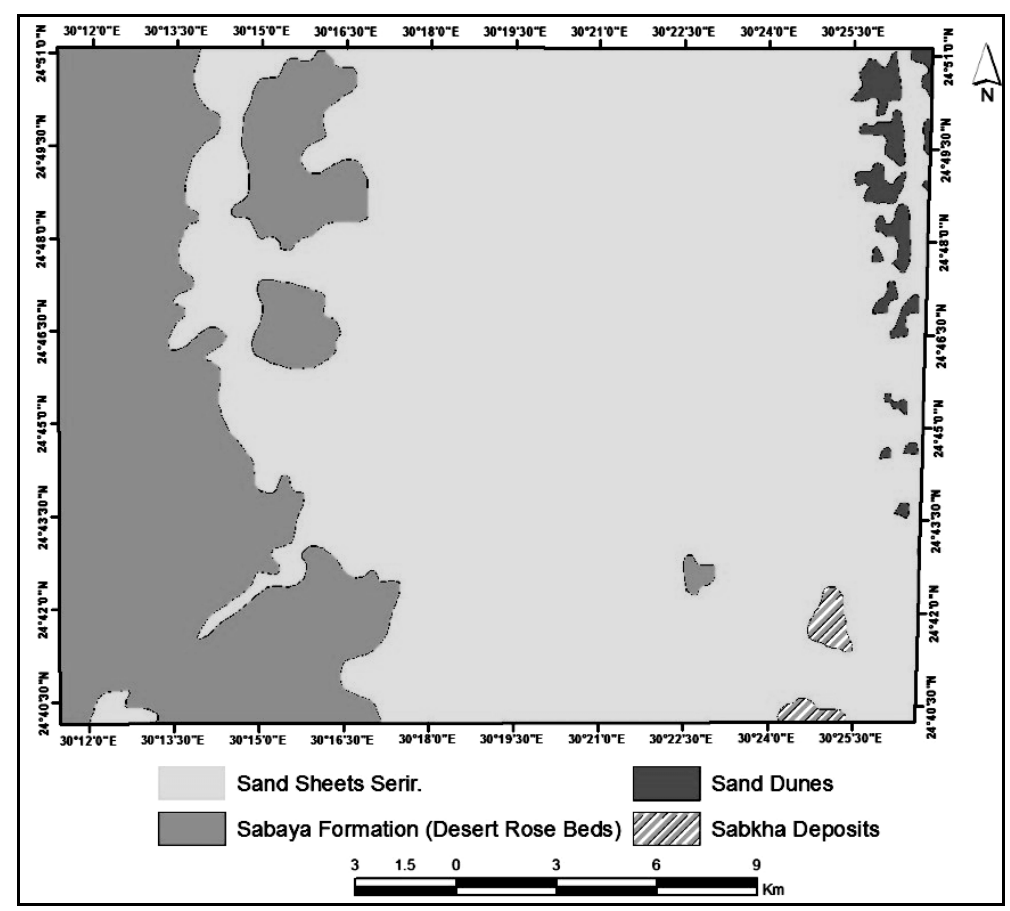

Figure 2. Geological map of the study area

Table 2. Geological formations of the study area (1: 500000)

\begin{tabular}{|l|c|c|}
\hline \multicolumn{1}{|c|}{ Geological Formation } & Area (feddan) & $\%$ \\
\hline Sand Sheets Serir. & 89158 & 71.92 \\
\hline Sabaya Formation (Desert Rose Beds) & 32515 & 26.23 \\
\hline Sand Dunes & 1668 & 1.35 \\
\hline Sabkha Deposits & 625 & 0.50 \\
\hline Total & 123966 & 100.0 \\
\hline
\end{tabular}

d) Geomorphology:

According to the geomorphological map (scale 1: 250000) produced by UNDPUNESCO (2005) the main form is Sand Sheets, which represents an area of about 84118 feddans $(67.86 \%)$ of the total study area followed by Pediplains covering an area of about 35798 feddans (28.88\%), while the Barchan Dunes Belts cover the rest 4050 feddans (3.26\%) (Figure 3 and Table 3). 


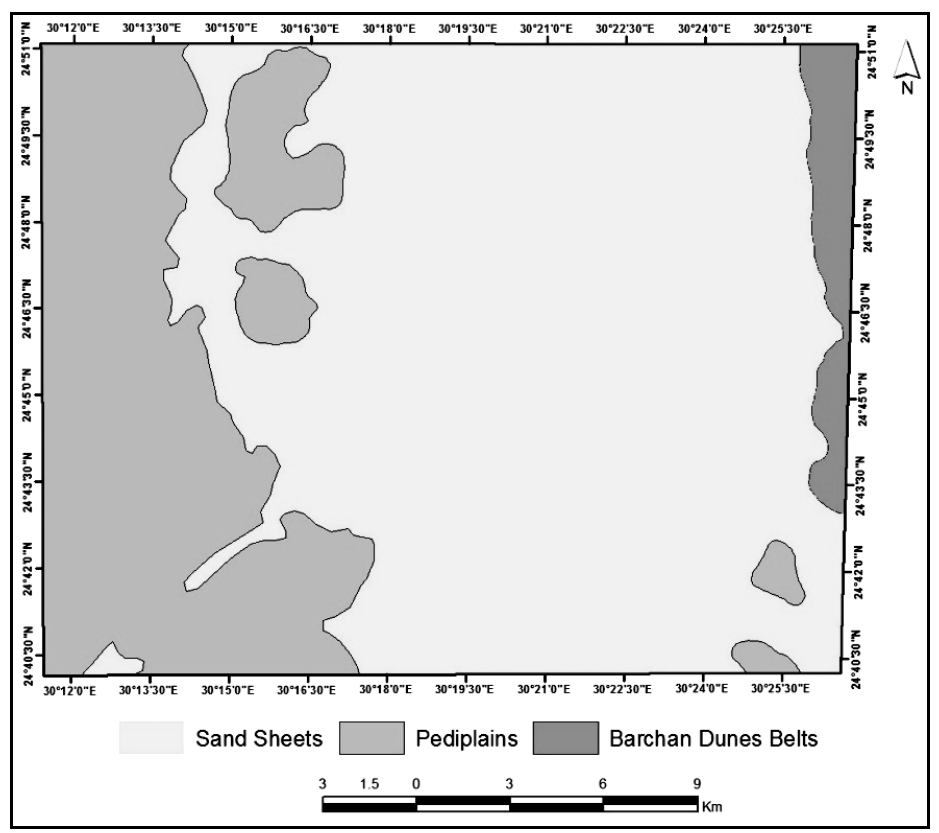

Figure 3. Geomorphological map of the study area

Table 3. Geomorphological forms of the study area (1: 250000)

\begin{tabular}{|l|c|c|}
\hline \multicolumn{1}{|c|}{ Geomorphological Form } & Area (feddan) & $\%$ \\
\hline Sand Sheets & 84118 & 67.86 \\
\hline Pediplains & 35798 & 28.88 \\
\hline Barchan Dunes Belts & 4050 & 3.26 \\
\hline Total & 123966 & 100.0 \\
\hline
\end{tabular}

e) Satellite data:

The data of landsat eight \{Landsat-8 image scene 176-43 (20/4/2014) with spatial resolution of $30 \mathrm{~m}$. and spectral resolution of the bands 5, 4 and 3$\}$ were used for delineating the physiographic units of the study area by the visual analysis, using the physiographic approach as proposed by Zinck (1988). This approach is based on the spectral signature of land features on the image. Image processing techniques were followed to produce the best possible enhanced image for visual interpretation. Spatial enhancement was done to have an output image with enhanced edges that related to soil. The pixel values are not manipulated individually but in relation to their four neighbors. This modifies the value of each pixel on neighboring brightness values (Daels, 1986). Colour enhancement was done to create new images from original in order to increase the amount of information that can be visually interpreted from the data.

The data and the output maps used the parameters for GIS displays were Egyptian Transverse Mercator projection (ETM) (Daels, 1986). 


\section{Field Work:}

Forty-three soil profiles were taken to represent the different mapping units of the study area. Twenty minipits were used for checking the boundaries between mapping units. Field work was done in Soil Survey Department and Remote Sensing Unit. Morphological descriptions were worked out for the soil profiles in the field according to FAO (2006) and classified according to the Soil Taxonomy System (USDA, 2010). The ground truth for the different physiographic units was conducted.

Soil representative samples of the different layers of soil profiles were taken for laboratory analyses

\section{Laboratory Analyses:}

The collected soil samples were air dried, crushed and prepared for laboratory analyses. Laboratory analyses were carried out for particle size distribution using the pipette method (Piper, 1950), calcium carbonate content using Collin's calcimeter (Black, 1982), gypsum content by precipitation with acetone and soil pH in the soil suspension 1:2.5 using $\mathrm{pH}$ meter and salinity as electrical conductivity (EC) in the soil paste extract (Jackson,1976).

\section{Building up Digital Georeference Database:}

The spatial data include vector data (shape files) use points and polygons to represent map features, while non spatial data include attributes information. The different soil attributes were coded and new fields were added and linked to the profile database file in Arc GIS 10.2 software. Each soil profile was geo-referenced using the Global Position Systems (GPS).

The following is an example of database of soil profiles and main chemical and physical properties as shown by Arc GIS 10.2 software.

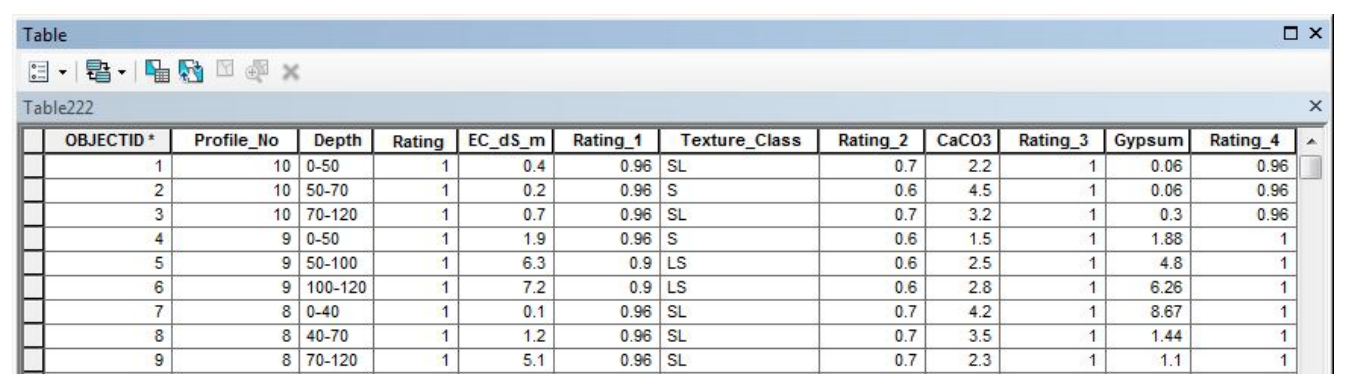

\section{Soil Units and Land Capability:}

Soils were categorized to the level of soil units according to Zinck (1988). Land evaluation for the purpose of the agricultural capability was assessed according to two methods:

Method 1: Storie Index (Storie, 1978) revised by O'Geen and Southard (2005) as a method for land evaluation according to the equation:

Storie index $=$ Factor $\mathrm{A} / 100 \times$ Factor $\mathrm{B} / 100 \times$ Factor $\mathrm{C} / 100 \times$ Factor $\mathrm{X} / 100 \times 100$ 
These factors are: (A) soil depth, (B) texture of the surface soil, (C) slope and (X) other factors or limitations (drainage and salts were taken as limiting factors in the study area). Each of these four general factors is evaluated on the basis of a "100 percent" rating. A rating of 100 percent expresses the most favorable, or ideal condition, and lower percentage ratings are given for conditions less favorable for crop production.

Capability grades classified according to the value of the index as follows:

\begin{tabular}{|l|l|l|}
\hline \multicolumn{1}{|c|}{ Grade } & \multicolumn{1}{c|}{ Index Rating } & \multicolumn{1}{c|}{ Definition } \\
\hline 1- Excellent & 80 through 100 & Soils are well suited to intensive use for growing irrigated crops. \\
\hline $2-$ Good & 60 through 79 & Soils are good agricultural soils. \\
\hline 3- Fair & 40 through 59 & $\begin{array}{l}\text { Soils are only fairly well suited to general agricultural use and } \\
\text { are limited. }\end{array}$ \\
\hline $4-$ Poor & 20 through 39 & $\begin{array}{l}\text { Soils are poorly suited. They are severely limited in their } \\
\text { agricultural potential. }\end{array}$ \\
\hline $5-$ Very Poor & 10 through 19 & Soils are very poorly suited for agriculture and seldom cultivated \\
\hline $6-$ Non & Less than 10 & $\begin{array}{l}\text { Soils are not suited for agriculture at all due to very severe to } \\
\text { extreme physical limitations. }\end{array}$ \\
\hline
\end{tabular}

Method 2: Land Capability techniques were done using the rating tables suggested by FAO (1985), Sys and Verheye (1978) and Sys et al. (1991) as common method for land evaluation according to the equation:

$$
C i=\frac{t}{100} \times \frac{w}{100} \times \frac{s_{1}}{100} \times \frac{s_{2}}{100} \times \frac{s_{3}}{100} \times \frac{s_{4}}{100} \times \frac{n}{100} \times 100
$$

Where:

$$
\begin{array}{ll}
\mathrm{Ci}=\text { Capability index (\%) } & \mathrm{S}_{2}=\text { Soil depth } \\
\mathrm{t}=\text { Slope } & \mathrm{S}_{3}=\mathrm{CaCO}_{3} \text { content } \\
\mathrm{w}=\text { Drainage conditions } & \mathrm{S}_{4}=\text { Gypsum content } \\
\mathrm{S}_{1}=\text { Texture } & \mathrm{n}=\text { Salinity and alkalinity }
\end{array}
$$

Capability classes arbitrary defined according to the value of the index as follows:

\begin{tabular}{|c|c|l|}
\hline Capability class & Land index $(\mathrm{Ci}) \%$ & \multicolumn{1}{|c|}{ Definition } \\
\hline S1 & $>75$ & Soils are highly suitable for cultivating all crops. \\
\hline S2 & $75-50$ & Soils are moderately suitable for agriculture \\
\hline S3 & $50-25$ & Soils are marginally suitable for agriculture \\
\hline N & $<25$ & Soils are not suitable for agriculture \\
\hline
\end{tabular}




\section{Land suitability assessment for specific crops.}

The assessment of land suitability for five different land use types (LUT) has been conducted for soil units using Sys et. al, (1993) by implementing the FAO Framework for Land Evaluation (FAO, 1976 b). Soil characteristics of the different mapping units were compared and matched with the requirements of each crop. The suitability maps were produced.

\section{RESULTS AND DISCUSSION}

\section{Physiographic soil map}

Visual interpretation was done on false colour composite of bands 5, 4, 3 scale 1:100000 to produce a base map according to the difference in landscape and relief for the field work activities (Zinck, 1988).

The integration between geology and geomorphology and visual interpretation was carried out to produce a base map. This base map was used in the field to check, confirm, correct and modify the physiographic mapping unit boundaries, coupled with the results of the field work to produce final physiographic soil map of the study area. Three landscape units were delineated, i.e. Plain (PI), Dunes (Du) and Hills (Hi) (Figure 4 and Table 4). The mapping unit of PI 111 belong to plain landscape unit, Du 111 belong to dunes landscape unit while $\mathrm{Hi} 111$ and $\mathrm{Hi}$ 112 belong to hills landscape unit. All mapping units are influenced by sandstone. The plain landscape unit is located in the eastern part of the study area. The area of this unit is about 83551 feddans ( $67.4 \%$ of the total study area) and contains one mapping unit, i.e. PI 111. The mapping unit of PI 111 was represented by 30 soil profiles. Dunes landscape unit represents small part adjacent to plain unit in eastern side of study area (4050 feddans $3.27 \%$ ). Hills landscape unit is located in western part of the study area. It represents an area of about 36365 feddans. (29.33\% of the total study area) and contains two mapping units i.e. Hi 111 and $\mathrm{Hi} 112$. The mapping unit $\mathrm{Hi} 112$ was represented by 13 soil profiles while $\mathrm{Hi} 111$ unit is out of soil profiles as rock lands. 


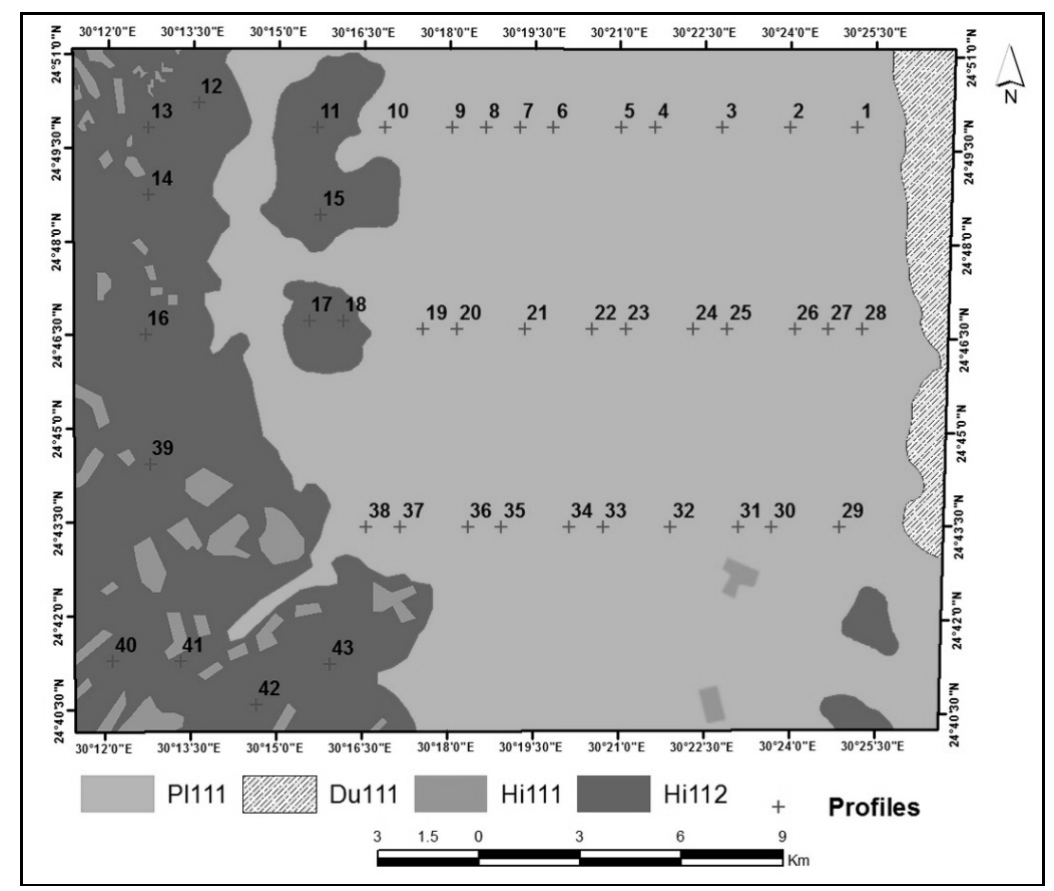

Figure 4. Location of soil profiles on Physiographic soil map of the study area

Table 4. Physiographic soil map legend

\begin{tabular}{|c|c|c|c|c|c|c|}
\hline \multirow[t]{2}{*}{$\begin{array}{c}\text { Landscape } \\
\text { Unit }\end{array}$} & \multirow{2}{*}{ Relief } & \multirow{2}{*}{ Lithology } & \multirow{2}{*}{ Landform } & \multirow[t]{2}{*}{$\begin{array}{l}\text { Mapping } \\
\text { Unit }\end{array}$} & \multicolumn{2}{|c|}{ Area } \\
\hline & & & & & feddan & $\%$ \\
\hline Plain (PI) & Flat (PI1) & Sandstone & Sand sheets & PI 111 & 83551 & 67.40 \\
\hline Dunes (Du) & Rolling (Du1) & Sandstone & $\begin{array}{c}\text { Barchan } \\
\text { dunes }\end{array}$ & Du 111 & 4050 & 3.27 \\
\hline \multirow[t]{2}{*}{ Hills (Hi) } & Low Hills (Hi1) & Sandstone & $\begin{array}{l}\text { Plateau } \\
\text { remnants }\end{array}$ & Hi 111 & 3553 & 2.87 \\
\hline & & Sandstone & Peidment & Hi 112 & 32812 & 26.46 \\
\hline Total & & & & & 123966 & 100 \\
\hline
\end{tabular}

The morphological description and taxonomic units of the obtained soil mapping units are summarized in Table (5).

\section{Soil Properties of mapping units.}

\subsection{Mapping Unit PI 111}

The soils of this unit are deep (120 cm in depth), the dominant texture is loamy sand, sandy loam and sand (clay fraction is between 2.0 and $18.5 \%$ ). Most of the surface soil layers are non-saline where the $\mathrm{EC} \mathrm{dS} / \mathrm{m}$ values are less than 4 while there is no clear trend for the different layers of the soil profiles. The soils are alkaline in reaction and not sodic as $\mathrm{pH}$ values are more than 7 and less than 8.5 in most areas. Exchangeable sodium percentage ranges between 5.7 and 12.9. Calcium carbonate content ranges from 1 to $13.2 \%$ except for areas effected by calcic horizon (Profiles $6,7,26$ and 28). Most values of gypsum content are less than $5 \%$ for surface layers (Table 6 ) except the surface layer of profile 8 where it reaches $8.7 \%$. 
Table 5. Morphological characteristics and taxonomic units of the studied area.

\begin{tabular}{|c|c|c|c|c|c|c|c|c|}
\hline $\begin{array}{l}\text { Mapping } \\
\text { Unit }\end{array}$ & Surface features & $\begin{array}{l}\text { Layer depth } \\
(\mathrm{cm})\end{array}$ & Texture & $\begin{array}{l}\text { Coarse } \\
\text { fragment }\end{array}$ & Structure & Consistency (dry) & Secondary formation & Taxonomic unit \\
\hline \multirow[t]{3}{*}{ PI 111} & \multirow{3}{*}{$\begin{array}{l}\text { Almost flat, covered } \\
\text { with thin sand } \\
\text { sheet, no } \\
\text { vegetation, } 100- \\
150 m \text { elevation } \\
\text { above sea level }\end{array}$} & $\begin{array}{l}\text { Surface up to } \\
25\end{array}$ & $\begin{array}{l}\text { Sand or } \\
\text { Loamy Sand }\end{array}$ & $\begin{array}{l}<5 \% \text { fine } \\
\text { gravel }\end{array}$ & $\begin{array}{l}\text { Single grains } \\
\text { or massive }\end{array}$ & Loose to soft & $\begin{array}{l}\text { Very few to common } \\
\text { soft gypsum }\end{array}$ & \multirow{3}{*}{$\begin{array}{l}\text { - Sandy - Sandy skeletal, } \\
\text { mixed families of Typic } \\
\text { Torriorthents and Sandy } \\
\text { mixed family of Typic } \\
\text { Haplocalcids associations. } \\
\text { - Fine - loamy, mixed family } \\
\text { of Leptic Haplogypsids as } \\
\text { inclusions. }\end{array}$} \\
\hline & & $\begin{array}{l}\text { Subsurface } \\
\text { up to } 60-75\end{array}$ & $\begin{array}{l}\text { Sand or } \\
\text { Loamy Sand }\end{array}$ & $\begin{array}{l}3 \text { to } 47 \% \text { fine } \\
\text { gravel }\end{array}$ & Massive & $\begin{array}{l}\text { Soft to slightly } \\
\text { hard }\end{array}$ & $\begin{array}{l}\text { Very few to common } \\
\text { soft lime }\end{array}$ & \\
\hline & & $\begin{array}{l}\text { Subsoil up to } \\
120\end{array}$ & $\begin{array}{l}\text { Sand or } \\
\text { Loamy Sand } \\
\text { or finer }\end{array}$ & $\begin{array}{l}<5 \% \text { fine } \\
\text { gravel }\end{array}$ & Massive & $\begin{array}{l}\text { Slightly hard to } \\
\text { hard }\end{array}$ & & \\
\hline Du 111 & \multicolumn{7}{|c|}{ High barchan sand dunes in rolling topographic, homogenous loose sand. } & $\begin{array}{l}\text { - Siliceous family of Typic } \\
\text { Torripsamments. }\end{array}$ \\
\hline Hi 111 & \multicolumn{7}{|c|}{ Plateau remnants rockland. } & - Rockland \\
\hline \multirow[t]{3}{*}{ Hi 112} & \multirow{3}{*}{$\begin{array}{l}\text { Gently undulating or } \\
\text { almost flat, locally } \\
\text { covered with stony } \\
\text { surface, no } \\
\text { vegetation } 150 \text { to } \\
175 \text { m elevation. }\end{array}$} & $\begin{array}{l}\text { Surface up to } \\
25\end{array}$ & $\begin{array}{l}\text { Sand or } \\
\text { loamy Sand }\end{array}$ & - & Massive & Soft & - & \multirow{3}{*}{$\begin{array}{l}\text { - Sand, mixed family of Typic - } \\
\text { Lithic Torriorthents } \\
\text { associations. } \\
\text { - Sand, mixed family of Typic } \\
\text { Hapocalcids as inclusions. }\end{array}$} \\
\hline & & $\begin{array}{l}\text { Subsurface } \\
\text { up to } 40-70\end{array}$ & $\begin{array}{l}\text { Sand or } \\
\text { loamy Sand }\end{array}$ & $\begin{array}{l}\text { Partly with } \\
\text { common } \\
\text { sandstone }\end{array}$ & Massive & Soft to hard & $\begin{array}{l}\text { Very few to common } \\
\text { soft lime }\end{array}$ & \\
\hline & & $40+$ to $70+$ & \multicolumn{5}{|l|}{ Sandstone. } & \\
\hline
\end{tabular}


Table 6. Chemical and physical properties of PI 111 mapping unit

\begin{tabular}{|c|c|c|c|c|c|c|c|c|c|c|c|}
\hline $\begin{array}{c}\text { Profile } \\
\text { No }\end{array}$ & Depth & $\begin{array}{c}\mathrm{pH} \\
(1: 2.5)\end{array}$ & $\begin{array}{c}E C \\
d S / m\end{array}$ & $\begin{array}{l}\mathrm{S}^{*} \\
\%\end{array}$ & $\begin{array}{l}\mathrm{SI} * \\
\%\end{array}$ & $\begin{array}{l}\text { C* } \\
\%\end{array}$ & $\begin{array}{l}\text { Texture } \\
\text { Class** }\end{array}$ & $\mathrm{CaCO}_{3}$ & Gypsum & ESP & $\begin{array}{c}\text { Gravel } \\
\% \\
\end{array}$ \\
\hline \multirow[t]{2}{*}{1} & $0-15$ & 7.8 & 2.2 & 92.0 & 2.2 & 5.8 & S & 13.2 & 0.7 & 8.3 & 4 \\
\hline & $15-120$ & 7.4 & 13.4 & 82.3 & 8.1 & 9.6 & LS & 2.0 & 0.1 & 11.1 & 36 \\
\hline \multirow[t]{2}{*}{2} & $0-20$ & 7.4 & 3.4 & 85.8 & 11.2 & 3.0 & LS & 1.6 & 3.5 & 8.4 & 3 \\
\hline & $20-120$ & 7.5 & 0.9 & 81.7 & 9.8 & 8.5 & LS & 2.5 & 1.7 & 5.7 & 42 \\
\hline \multirow[t]{3}{*}{3} & $0-20$ & 7.7 & 9.7 & 93.1 & 4.9 & 2.0 & S & 1.4 & 2.5 & 6.3 & 4 \\
\hline & $20-60$ & 7.9 & 7.6 & 83.8 & 11.5 & 4.7 & LS & 5.6 & 1.8 & 7.7 & 37 \\
\hline & $60-120$ & 7.4 & 8.6 & 92.6 & 3.9 & 3.5 & $\mathrm{~S}$ & 1.4 & 2.5 & 9.3 & 3 \\
\hline \multirow[t]{3}{*}{4} & $0-30$ & 7.5 & 0.8 & 75.5 & 10.5 & 14.0 & $\mathrm{SL}$ & 4.9 & 2.5 & 7.5 & 17 \\
\hline & $30-70$ & 7.7 & 3.0 & 76.7 & 9.6 & 13.7 & SL & 5.6 & 3.3 & 7.9 & 40 \\
\hline & $70-120$ & 7.7 & 3.2 & 79.0 & 13.1 & 7.9 & SL & 4.2 & 2.6 & 8.3 & 17 \\
\hline \multirow[t]{3}{*}{5} & $0-30$ & 7.6 & 4.5 & 75.0 & 11.0 & 14.0 & SL & 1.9 & 5.9 & 7.9 & 9 \\
\hline & $30-50$ & 7.8 & 7.1 & 73.0 & 14.0 & 13.0 & SL & 1.9 & 5.9 & 6.3 & 42 \\
\hline & $50-120$ & 7.8 & 13.2 & 61.5 & 21.8 & 16.7 & SL & 2.3 & 4.3 & 9.2 & 4 \\
\hline \multirow[t]{3}{*}{6} & $0-25$ & 8.0 & 0.8 & 85.0 & 12.4 & 2.6 & LS & 5.4 & 3.4 & 10.2 & 13 \\
\hline & $25-50$ & 7.6 & 6.1 & 75.3 & 11.7 & 13.0 & SL & 17.5 & 4.4 & 11.2 & 6 \\
\hline & $50-120$ & 7.5 & 6.0 & 77.0 & 10.5 & 12.5 & SL & 5.9 & 3.7 & 12.1 & 6 \\
\hline \multirow[t]{3}{*}{7} & $0-20$ & 7.4 & 1.8 & 91.6 & 5.7 & 2.7 & $\mathrm{~s}$ & 9.1 & 3.3 & 8.3 & 17 \\
\hline & $20-40$ & 7.5 & 6.3 & 85.8 & 11.5 & 2.7 & LS & 16.9 & 2.4 & 9.2 & 7 \\
\hline & $40-120$ & 7.5 & 7.3 & 86.0 & 10.7 & 3.3 & LS & 11.2 & 3.1 & 10.3 & 10 \\
\hline \multirow[t]{3}{*}{8} & $0-40$ & 7.4 & 0.1 & 72.9 & 17.6 & 9.5 & SL & 4.2 & 8.7 & 11.1 & 23 \\
\hline & $40-70$ & 8.4 & 1.2 & 72.9 & 17.6 & 9.5 & SL & 3.5 & 1.4 & 7.9 & 44 \\
\hline & $70-120$ & 8.0 & 5.1 & 75.3 & 11.7 & 13.0 & SL & 2.3 & 1.1 & 8.5 & 25 \\
\hline \multirow[t]{3}{*}{9} & $0-50$ & 8.2 & 1.9 & 93.1 & 4.9 & 2.0 & $\mathrm{~s}$ & 1.5 & 1.9 & 9.3 & 30 \\
\hline & $50-100$ & 7.9 & 6.3 & 83.8 & 11.5 & 4.7 & LS & 2.5 & 4.8 & 10.2 & 47 \\
\hline & $100-120$ & 7.9 & 7.2 & 85.8 & 11.2 & 3.0 & LS & 2.8 & 6.3 & 11.3 & 40 \\
\hline \multirow[t]{3}{*}{10} & $0-50$ & 7.6 & 0.4 & 72.9 & 17.6 & 9.5 & SL & 2.2 & 0.1 & 12.9 & 30 \\
\hline & $50-70$ & 7.4 & 0.2 & 91.4 & 5.7 & 2.9 & $\mathrm{~s}$ & 4.5 & 0.1 & 10.3 & 40 \\
\hline & $70-120$ & 7.8 & 0.7 & 76.2 & 10.1 & 13.7 & SL & 3.2 & 0.3 & 10.5 & 20 \\
\hline \multirow[t]{3}{*}{19} & $0-20$ & 7.5 & 0.8 & 84.6 & 11.4 & 4.0 & LS & 4.5 & 0.1 & 10.1 & 4 \\
\hline & $20-50$ & 7.4 & 0.9 & 85.1 & 10.9 & 4.0 & LS & 1.8 & 0.2 & 9.3 & 47 \\
\hline & 50-120 & 7.9 & 1.1 & 90.0 & 6.6 & 3.4 & $\mathrm{~s}$ & 5.3 & 0.2 & 9.2 & 4 \\
\hline \multirow[t]{3}{*}{20} & $0-20$ & 7.8 & 0.4 & 90.5 & 6.9 & 2.6 & $\mathrm{~s}$ & 10.0 & 0.2 & 9.0 & 3 \\
\hline & $20-80$ & 7.3 & 3.8 & 94.7 & 2.5 & 2.8 & $\mathrm{~S}$ & 2.0 & 0.2 & 8.7 & 46 \\
\hline & $80-120$ & 7.8 & 3.1 & 92.2 & 4.3 & 3.5 & $\mathrm{~s}$ & 7.5 & 0.5 & 7.6 & 5 \\
\hline \multirow[t]{2}{*}{21} & $0-20$ & 7.7 & 0.3 & 93.8 & 3.5 & 2.7 & $\mathrm{~s}$ & 2.7 & 2.7 & 8.7 & 4 \\
\hline & $20-120$ & 7.4 & 3.3 & 86.1 & 10.6 & 3.3 & LS & 3.8 & 1.3 & 10.2 & 44 \\
\hline \multirow[t]{2}{*}{22} & $0-40$ & 8.1 & 5.0 & 85.2 & 11.0 & 3.8 & LS & 11.2 & 3.0 & 10.3 & 3 \\
\hline & $40-120$ & 8.0 & 9.5 & 82.4 & 15.0 & 2.6 & $\mathrm{~s}$ & 5.6 & 2.7 & 10.0 & 33 \\
\hline \multirow[t]{2}{*}{23} & $0-45$ & 7.8 & 1.8 & 79.4 & 15.0 & 5.6 & LS & 6.3 & 4.0 & 10.1 & 4 \\
\hline & $45-120$ & 7.9 & 2.3 & 85.3 & 11.2 & 3.5 & LS & 7.1 & 3.2 & 9.5 & 47 \\
\hline \multirow[t]{2}{*}{24} & $0-20$ & 7.7 & 0.3 & 85.1 & 10.9 & 4.0 & LS & 10.5 & 1.5 & 11.2 & 5 \\
\hline & $20-120$ & 7.4 & 3.3 & 89.0 & 6.4 & 4.6 & LS & 5.6 & 1.4 & 11.3 & 37 \\
\hline 25 & $0-15$ & 8.1 & 1.7 & 90.0 & 5.1 & 4.9 & $\mathrm{~S}$ & 3.0 & 0.2 & 11.2 & 4 \\
\hline
\end{tabular}

$* \mathrm{~S}=$ Sand, $\mathrm{SI}=$ Slit and $\mathrm{C}=$ Clay

** S= Sand, LS = Loamy Sand, SL=Sandy Loam, SCL = Sandy Clay Loam, CL= Clay Loam and C= Clay 
Table 6. cont.

\begin{tabular}{|c|c|c|c|c|c|c|c|c|c|c|c|}
\hline $\begin{array}{l}\text { Profile } \\
\text { No }\end{array}$ & Depth & $\begin{array}{c}\mathrm{pH} \\
(1: 2.5) \\
\end{array}$ & $\begin{array}{r}\mathrm{EC} \\
\mathrm{dS} / \mathrm{m} \\
\end{array}$ & $\begin{array}{l}\mathrm{S}^{*} \\
\% \\
\end{array}$ & $\begin{array}{l}\text { SI* } \\
\%\end{array}$ & $\begin{array}{l}\text { C* } \\
\% \\
\end{array}$ & $\begin{array}{l}\text { Texture } \\
\text { Class** }\end{array}$ & $\mathrm{CaCO}_{3}$ & Gypsum & ESP & $\begin{array}{c}\text { Gravel } \\
\% \\
\end{array}$ \\
\hline & $15-120$ & 7.6 & 10.6 & 87.8 & 7.4 & 4.8 & LS & 2.5 & 1.1 & 10.3 & 37 \\
\hline \multirow[t]{3}{*}{26} & $0-20$ & 7.6 & 8.5 & 90.3 & 6.6 & 3.1 & $\mathrm{~s}$ & 6.2 & 1.1 & 12.3 & 5 \\
\hline & $20-60$ & 7.7 & 9.6 & 83.7 & 10.0 & 6.3 & LS & 16.5 & 5.2 & 11.5 & 35 \\
\hline & $60-120$ & 7.8 & 5.3 & 76.2 & 18.2 & 5.6 & LS & 5.1 & 3.2 & 11.2 & 4 \\
\hline \multirow[t]{2}{*}{27} & $0-60$ & 7.5 & 0.7 & 61.5 & 21.8 & 16.7 & SL & 1.9 & 0.1 & 12.1 & 5 \\
\hline & $60-120$ & 7.7 & 1.2 & 61.5 & 21.8 & 16.7 & SL & 2.0 & 0.3 & 10.9 & 37 \\
\hline \multirow[t]{3}{*}{28} & $0-30$ & 7.5 & 0.5 & 79.3 & 15.1 & 5.6 & LS & 5.5 & 2.1 & 10.7 & 5 \\
\hline & $30-60$ & 7.5 & 0.1 & 77.3 & 13.4 & 9.3 & SL & 16.9 & 1.9 & 10.6 & 7 \\
\hline & $60-120$ & 7.5 & 0.1 & 83.1 & 13.3 & 3.6 & LS & 5.6 & 0.1 & 10.5 & 3 \\
\hline \multirow[t]{3}{*}{29} & $0-20$ & 7.9 & 4.3 & 69.2 & 13.3 & 17.5 & SL & 11.0 & 1.0 & 12.3 & 3 \\
\hline & $20-60$ & 7.9 & 14.4 & 30.3 & 36.4 & 33.3 & $\mathrm{CL}$ & 3.5 & 5.2 & 11.7 & 2 \\
\hline & $60-120$ & 7.5 & 15.2 & 70.3 & 11.2 & 18.5 & SL & 2.0 & 5.0 & 11.3 & 3 \\
\hline \multirow[t]{3}{*}{30} & $0-50$ & 7.4 & 12.8 & 49.0 & 29.5 & 21.5 & $\mathrm{SCL}$ & 5.7 & 6.9 & 12.1 & 4 \\
\hline & 50-75 & 7.9 & 15.2 & 35.0 & 31.0 & 34.0 & $\mathrm{CL}$ & 3.1 & 0.1 & 9.9 & 2 \\
\hline & $75-120$ & 7.6 & 7.7 & 44.0 & 20.0 & 36.0 & $C$ & 5.4 & 1.9 & 9.9 & 2 \\
\hline \multirow[t]{2}{*}{31} & $0-25$ & 7.7 & 6.0 & 64.1 & 17.0 & 18.9 & SL & 3.0 & 0.2 & 11.3 & 4 \\
\hline & $25-120$ & 8.3 & 14.5 & 45.0 & 14.5 & 40.5 & C & 5.5 & 2.1 & 11.5 & 5 \\
\hline \multirow[t]{2}{*}{32} & $0-15$ & 7.2 & 0.5 & 85.1 & 11.1 & 3.8 & LS & 1.6 & 1.5 & 10.7 & 4 \\
\hline & $15-120$ & 7.4 & 4.1 & 55.7 & 16.5 & 27.8 & $\mathrm{SCL}$ & 1.7 & 1.2 & 9.9 & 35 \\
\hline \multirow[t]{4}{*}{33} & $0-20$ & 7.7 & 0.6 & 93.1 & 4.9 & 2.0 & $\mathrm{~s}$ & 2.2 & 2.5 & 8.9 & 4 \\
\hline & $20-40$ & 7.7 & 2.2 & 86.9 & 9.5 & 3.6 & $\mathrm{~S}$ & 2.7 & 3.2 & 10.5 & 40 \\
\hline & $40-70$ & 7.9 & 3.0 & 85.2 & 10.0 & 4.8 & LS & 2.8 & 8.3 & 12.3 & 37 \\
\hline & $70-120$ & 7.7 & 3.8 & 85.8 & 10.2 & 4.0 & LS & 1.3 & 5.4 & 12.5 & 5 \\
\hline \multirow[t]{3}{*}{34} & $0-20$ & 7.6 & 5.0 & 86.2 & 9.8 & 4.0 & LS & 4.5 & 2.5 & 12.1 & 6 \\
\hline & $20-40$ & 7.7 & 5.0 & 85.1 & 11.7 & 3.2 & $\mathrm{~S}$ & 3.5 & 3.9 & 11.9 & 35 \\
\hline & $40-120$ & 8.1 & 14.3 & 87.3 & 8.5 & 4.2 & LS & 2.1 & 2.4 & 11.3 & 4 \\
\hline \multirow[t]{3}{*}{35} & $0-20$ & 7.8 & 0.9 & 77.8 & 10.9 & 11.3 & SL & 2.0 & 1.5 & 10.3 & 4 \\
\hline & $20-40$ & 7.9 & 7.3 & 63.0 & 20.0 & 17.0 & SL & 2.0 & 1.6 & 10.6 & 40 \\
\hline & $40-120$ & 7.7 & 9.1 & 64.7 & 19.0 & 16.3 & SL & 1.8 & 1.3 & 10.6 & 3 \\
\hline \multirow[t]{3}{*}{36} & $0-20$ & 7.9 & 2.7 & 86.2 & 9.8 & 4.0 & LS & 3.5 & 2.8 & 11.3 & 6 \\
\hline & $20-40$ & 8.0 & 4.2 & 83.1 & 13.3 & 3.6 & LS & 4.2 & 1.6 & 11.2 & 44 \\
\hline & $40-120$ & 8.0 & 4.6 & 84.5 & 10.9 & 4.6 & LS & 1.8 & 2.2 & 10.8 & 6 \\
\hline \multirow[t]{3}{*}{37} & $0-30$ & 8.0 & 1.8 & 85.6 & 11.4 & 3.0 & LS & 7.7 & 0.1 & 10.1 & 5 \\
\hline & $30-80$ & 7.7 & 1.4 & 49.7 & 24.8 & 25.5 & $\mathrm{SCL}$ & 1.7 & 9.0 & 10.6 & 20 \\
\hline & $80-120$ & 7.4 & 1.3 & 41.1 & 19.7 & 39.2 & $\mathrm{C}$ & 1.3 & 8.0 & 10.9 & 4 \\
\hline \multirow[t]{2}{*}{38} & $0-30$ & 7.7 & 0.6 & 85.8 & 10.9 & 3.3 & LS & 1.0 & 0.1 & 11.4 & 3 \\
\hline & $30-120$ & 7.9 & 1.1 & 85.8 & 10.2 & 4.0 & LS & 4.4 & 0.2 & 11.2 & 36 \\
\hline
\end{tabular}

\footnotetext{
* $\mathrm{S}=$ Sand, $\mathrm{SI}=$ Slit and $\mathrm{C}=$ Clay
}

** S= Sand, LS = Loamy Sand, SL=Sandy Loam, SCL = Sandy Clay Loam, CL= Clay Loam and C= Clay

\subsection{Mapping Unit Hi 112}

The soils of this unit are moderately deep (50-100 cm in depth) except for profiles 18,39 and 41 where the soils are shallow to very shallow. The dominant texture is sand and loamy sand (clay fraction is between 2.0 and $4.8 \%$ ). The surface layers are saline where values of EC dS/m are more than 4 except for profiles 12,13 , and 14 (EC values are less than $2 \mathrm{dS} / \mathrm{m}$ ). The soils are alkaline as $\mathrm{pH}$ values are more than 7 while, exchangeable sodium percentage ranges between 9.2 and 12.4. Calcium carbonate content ranges between 0.4 and $13.3 \%$. Gypsum content less $2.1 \%$ (Table 7). 
Table 7. Chemical and physical properties of Hi 112 mapping unit

\begin{tabular}{|c|c|c|c|c|c|c|c|c|c|c|c|}
\hline $\begin{array}{c}\text { Profile } \\
\text { NO }\end{array}$ & Depth & $\begin{array}{c}\mathrm{pH} \\
(1: 2.5)\end{array}$ & $\begin{array}{c}E C \\
d S / m\end{array}$ & $\begin{array}{l}\text { S* } \\
\%\end{array}$ & $\begin{array}{l}\mathrm{Sl} * \\
\%\end{array}$ & $\begin{array}{l}\text { C* } \\
\% \\
\end{array}$ & $\begin{array}{l}\text { Texture } \\
\text { Class** }\end{array}$ & $\mathrm{CaCO}_{3}$ & Gypsum & ESP & $\begin{array}{c}\text { Gravel } \\
\% \\
\end{array}$ \\
\hline \multirow[t]{2}{*}{11} & $0-50$ & 8.2 & 7.4 & 86.5 & 10.6 & 2.9 & $\mathrm{~S}$ & 10.5 & 5.1 & 10.9 & 4 \\
\hline & $50-60$ & 7.9 & 14.3 & 86.5 & 11.0 & 2.5 & $\mathrm{~s}$ & 3.6 & 0.9 & 11.1 & 3 \\
\hline \multirow[t]{3}{*}{12} & $0-15$ & 7.7 & 0.9 & 75.3 & 11.7 & 13.0 & SL & 4.6 & 0.1 & 9.2 & 2 \\
\hline & $15-50$ & 8.1 & 12.5 & 77.0 & 10.5 & 12.5 & SL & 9.8 & 2.0 & 11.5 & 3 \\
\hline & $50-60$ & 7.9 & 20.2 & 84.4 & 11.4 & 4.2 & LS & 7.7 & 2.0 & 11.6 & 3 \\
\hline \multirow[t]{2}{*}{13} & $0-40$ & 7.6 & 0.5 & 93.1 & 4.9 & 2.0 & $\mathrm{~S}$ & 6.8 & 0.3 & 11.2 & 2 \\
\hline & $40-100$ & 7.3 & 10.0 & 87.5 & 9.6 & 2.9 & $\mathrm{~S}$ & 5.2 & 0.1 & 10.3 & 4 \\
\hline \multirow[t]{3}{*}{14} & $0-20$ & 8.1 & 0.4 & 91.2 & 4.3 & 4.5 & $\mathrm{~S}$ & 4.9 & 2.1 & 12.3 & 1 \\
\hline & $20-40$ & 8.0 & 19.0 & 91.9 & 4.1 & 4.0 & $\mathrm{~S}$ & 3.8 & 1.7 & 12.4 & 3 \\
\hline & $40-60$ & 8.0 & 29.0 & 92.7 & 4.6 & 2.7 & $\mathrm{~s}$ & 3.6 & 5.0 & 11.5 & 3 \\
\hline \multirow[t]{2}{*}{15} & $0-20$ & 7.5 & 32.0 & 88.8 & 6.4 & 4.8 & LS & 3.5 & 0.1 & 10.5 & 2 \\
\hline & $20-60$ & 7.7 & 31.7 & 75.2 & 11.5 & 13.3 & SL & 7.9 & 0.2 & 10.6 & 4 \\
\hline \multirow[t]{3}{*}{16} & $0-30$ & 7.5 & 5.3 & 86.7 & 10.6 & 2.7 & $\mathrm{~S}$ & 13.3 & 5.2 & 10.9 & 2 \\
\hline & $30-70$ & 7.5 & 6.1 & 89.8 & 4.4 & 5.8 & $\mathrm{~S}$ & 4.8 & 0.2 & 11.2 & 4 \\
\hline & $70-100$ & 7.5 & 5.4 & 87.3 & 8.5 & 4.2 & $S$ & 0.4 & 0.2 & 10.9 & 2 \\
\hline \multirow[t]{2}{*}{17} & $0-35$ & 7.9 & 6.6 & 91.0 & 5.0 & 4.0 & $\mathrm{~S}$ & 1.4 & 2.1 & 10.3 & 1 \\
\hline & $35-100$ & 8.1 & 4.3 & 84.5 & 11.8 & 3.7 & LS & 4.4 & 0.8 & 11.2 & 3 \\
\hline \multirow[t]{2}{*}{18} & $0-30$ & 7.7 & 5.6 & 66.3 & 17.8 & 15.9 & SL & 3.5 & 1.4 & 10.3 & 1 \\
\hline & $30-45$ & 8.0 & 4.8 & 90.4 & 4.3 & 5.3 & $S$ & 7.7 & 1.5 & 10.9 & 3 \\
\hline 39 & $0-45$ & 7.8 & 7.1 & 92.2 & 3.5 & 4.3 & $S$ & 2.1 & 1.8 & 11.2 & 3 \\
\hline \multirow[t]{3}{*}{40} & $0-25$ & 7.7 & 5.9 & 84.2 & 13.2 & 2.6 & LS & 3.5 & 2.0 & 11.5 & 1 \\
\hline & $25-65$ & 7.8 & 7.0 & 88.4 & 8.5 & 3.1 & LS & 6.3 & 2.3 & 11.4 & 4 \\
\hline & $65-90$ & 7.8 & 5.7 & 88.7 & 8.0 & 3.3 & LS & 7.0 & 3.5 & 10.3 & 3 \\
\hline 41 & $0-25$ & 7.6 & 7.2 & 89.9 & 7.6 & 2.5 & $S$ & 1.4 & 1.7 & 10.5 & 4 \\
\hline \multirow[t]{3}{*}{42} & $0-25$ & 7.9 & 7.3 & 86.1 & 11.0 & 2.9 & LS & 2.6 & 2.0 & 11.6 & 2 \\
\hline & $25-40$ & 7.6 & 6.1 & 67.7 & 16.7 & 15.6 & SL & 4.3 & 0.1 & 11.2 & 4 \\
\hline & $40-60$ & 8.1 & 6.1 & 63.9 & 19.5 & 16.6 & SL & 5.2 & 0.1 & 10.9 & 3 \\
\hline \multirow[t]{2}{*}{43} & $0-25$ & 7.9 & 4.1 & 85.3 & 12.7 & 2.0 & LS & 1.5 & 1.5 & 10.6 & 2 \\
\hline & $25-60$ & 7.7 & 6.3 & 85.5 & 10.5 & 4.0 & LS & 2.3 & 2.1 & 11.3 & 4 \\
\hline
\end{tabular}

$* \mathrm{~S}=$ Sand, $\mathrm{Sl}=$ Slit and C = Clay

** S = Sand, LS = Loamy Sand and SL=Sandy Loam 


\section{Land capability assessment}

A land capability model was built using Arc GIS 10.2 software (database) and the resulting tables were imported into Arc GIS to produce the capability map. The soils of the studied area were classified according two methods:

Method 1: Based on the Storie Index model as shown in Figure (5) could be classified into three capability grades reflecting the limitation factor, i.e. grade 1 , grade 3 and grade 6 . The soils of grade 1 have almost no limitation factors for agricultural crops. It represent an area of about 83551 feddans $(67.4 \%$ of the total area). The soils of grade3, whereas soil depth and salinity are the main limiting factors, occupies an area of 32432 feddans (26.16\%). While the grade 6 occupies 7983 feddans ( $6.44 \%$ of the total study) area including the areas of sand dunes, rockland and shallow to very shallow soils.

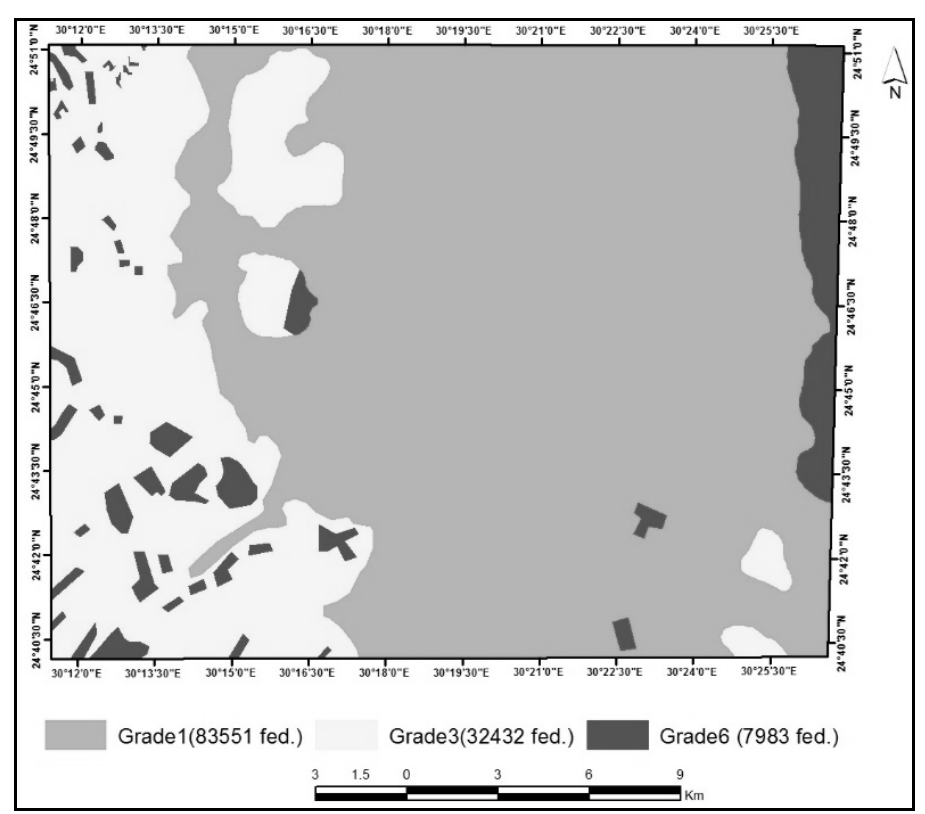

Figure 5. Land capability map of the study area according Storie Index

Method 2: Based on the Sys model as shown in Figure (6) was classified into three capability classes which reflect the limitation factors, i.e. $S_{2}, S_{3}$ and $N_{2}$. The soils of $S_{2}$ have moderate limitations for agricultural crops, as texture is the main limiting factor with area 83551 feddans ( $67.4 \%$ of the total area). The soils of $S_{3}$ where texture, depth and salinity are the main limiting factors, occupies an area of 32432 feddans (26.16\%), while the $\mathrm{N}_{2}$ occupied 7983 feddans (6.44\% of the total study area) including the areas of sand dunes, rockland and very shallow soils. 


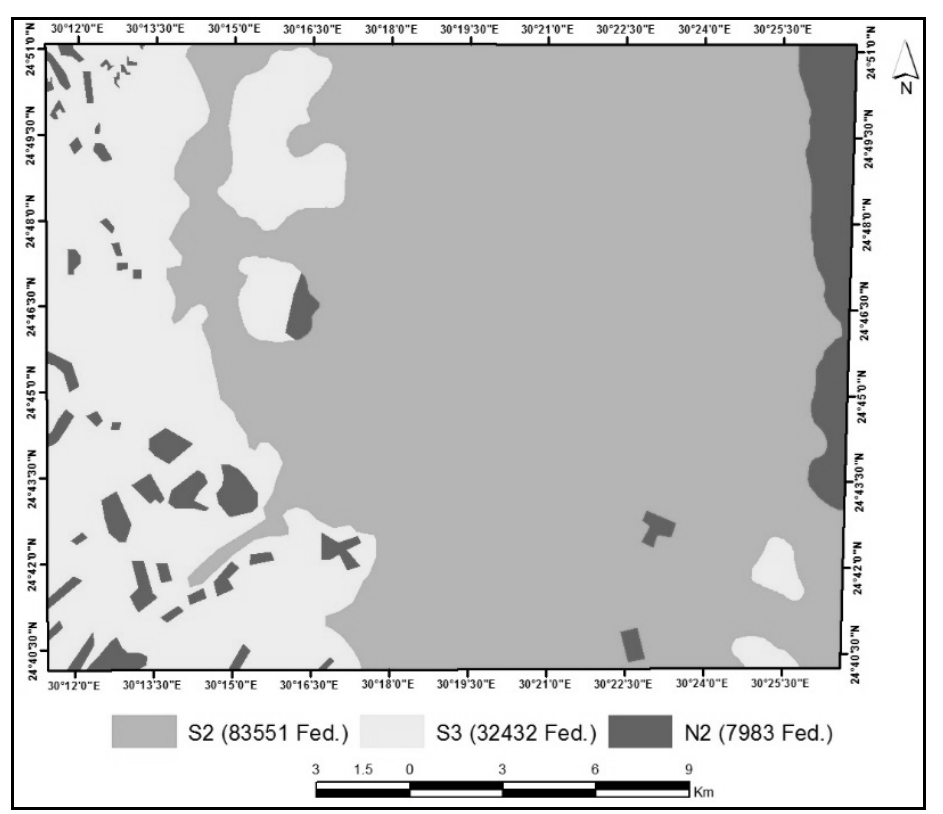

Figure 6. Land capability map of the study area according Sys model

The results of land capability indicate that capability degrees of Storie Index were grade 1 with an area of 83551 feddans (67.4\%), grade 3 occupies an area of about 32432 feddans ( $26.16 \%$ ) and grade 6 occupies 7983 feddans $(6.44 \%)$ that is equal to the capability classes of Sys method $S_{2}, S_{3}$, and $N_{2}$ with same areas, respectively. The capability index and rating of main characteristics for mapping units (Storie, 1978) are as follows :

\begin{tabular}{|c|c|c|c|c|c|c|c|c|}
\hline Unit & Profile No & Depth & Texture & Slope & EC & Drainage & ${ }^{*}$ Ci $\%$ & Grade \\
\hline PI 111 & 1 & 1 & 0.92 & 1 & 0.91 & 1 & 83.7 & Grade 1 \\
\hline & 2 & 1 & 0.92 & 1 & 0.95 & 1 & 87.4 & Grade 1 \\
\hline & 3 & 1 & 0.92 & 1 & 0.9 & 1 & 82.8 & Grade 1 \\
\hline & 4 & 1 & 0.92 & 1 & 0.95 & 1 & 87.4 & Grade 1 \\
\hline & 5 & 1 & 0.92 & 1 & 0.91 & 1 & 83.7 & Grade 1 \\
\hline & 7 & 1 & 0.92 & 1 & 0.91 & 1 & 83.7 & Grade 1 \\
\hline & 8 & 1 & 0.92 & 1 & 0.95 & 1 & 87.4 & Grade 1 \\
\hline & 9 & 1 & 0.92 & 1 & 0.95 & 1 & 87.4 & Grade 1 \\
\hline & 10 & 1 & 0.92 & 1 & 0.95 & 1 & 87.4 & Grade 1 \\
\hline & 19 & 1 & 0.92 & 1 & 0.95 & 1 & 87.4 & Grade 1 \\
\hline & 20 & 1 & 0.92 & 1 & 0.95 & 1 & 87.4 & Grade 1 \\
\hline & 21 & 1 & 0.92 & 1 & 0.95 & 1 & 87.4 & Grade 1 \\
\hline & 22 & 1 & 0.92 & 1 & 0.95 & 1 & 87.4 & Grade 1 \\
\hline
\end{tabular}

${ }^{*} \mathrm{Ci}=$ Capability index 
Cont.

\begin{tabular}{|c|c|c|c|c|c|c|c|c|}
\hline Unit & Profile No & Depth & Texture & Slope & EC & Drainage & $* \mathrm{Ci} \%$ & Grade \\
\hline & 23 & 1 & 0.92 & 1 & 0.95 & 1 & 87.4 & grade 1 \\
\hline & 24 & 1 & 0.92 & 1 & 0.95 & 1 & 87.4 & grade 1 \\
\hline & 25 & 1 & 0.92 & 1 & 0.91 & 1 & 83.7 & grade 1 \\
\hline & 26 & 1 & 0.92 & 1 & 0.91 & 1 & 83.7 & grade 1 \\
\hline & 27 & 1 & 0.92 & 1 & 0.95 & 1 & 87.4 & grade 1 \\
\hline & 28 & 1 & 0.92 & 1 & 0.95 & 1 & 87.4 & grade 1 \\
\hline & 29 & 1 & 0.92 & 1 & 0.85 & 1 & 78.2 & grade 1 \\
\hline & 30 & 1 & 0.92 & 1 & 0.85 & 1 & 78.2 & grade 1 \\
\hline & 31 & 1 & 0.92 & 1 & 0.91 & 1 & 83.7 & grade 1 \\
\hline & 32 & 1 & 0.92 & 1 & 0.91 & 1 & 83.7 & grade 1 \\
\hline & 33 & 1 & 0.92 & 1 & 0.91 & 1 & 83.7 & grade 1 \\
\hline & 34 & 1 & 0.92 & 1 & 0.91 & 1 & 83.7 & grade 1 \\
\hline & 35 & 1 & 0.92 & 1 & 0.95 & 1 & 87.4 & grade 1 \\
\hline & 36 & 1 & 0.92 & 1 & 0.9 & 1 & 82.8 & grade 1 \\
\hline & 37 & 1 & 0.92 & 1 & 0.95 & 1 & 87.4 & grade 1 \\
\hline & 38 & 1 & 0.92 & 1 & 0.95 & 1 & 87.4 & grade 1 \\
\hline Hi 112 & 11 & 0.7 & 0.92 & 1 & 0.82 & 0.96 & 50.7 & grade 3 \\
\hline & 12 & 0.7 & 0.92 & 1 & 0.82 & 0.96 & 50.7 & grade 3 \\
\hline & 13 & 0.7 & 0.92 & 1 & 0.82 & 0.96 & 50.7 & grade 3 \\
\hline & 14 & 0.7 & 0.92 & 1 & 0.7 & 0.96 & 43.3 & grade 3 \\
\hline & 15 & 0.7 & 0.92 & 1 & 0.7 & 0.96 & 43.3 & grade 3 \\
\hline & 16 & 0.7 & 0.92 & 1 & 0.82 & 0.96 & 50.7 & grade 3 \\
\hline & 17 & 0.7 & 0.92 & 1 & 0.82 & 0.96 & 50.7 & grade 3 \\
\hline & 18 & - & - & - & - & - & - & grade 6 \\
\hline & 39 & - & - & - & - & - & - & grade 6 \\
\hline & 40 & 0.7 & 0.92 & 1 & 0.8 & 0.96 & 49.5 & grade 3 \\
\hline & 41 & _ & - & _ & _ & _ & _ & grade 6 \\
\hline & 42 & 0.7 & 0.92 & 1 & 0.8 & 0.96 & 49.5 & grade 3 \\
\hline & 43 & 0.7 & 0.92 & 1 & 0.8 & 0.96 & 49.5 & grade 3 \\
\hline Hi 112 & - & - & - & - & - & - & - & grade 6 \\
\hline Du 111 & - & - & - & - & - & - & - & grade 6 \\
\hline
\end{tabular}

${ }^{*} \mathrm{Ci}=$ Capability index 
In addition, the capability index and rating of main characteristics for mapping units according to Sys (1991) are as follows:

\begin{tabular}{|c|c|c|c|c|c|c|c|c|c|c|}
\hline Unit & $\begin{array}{l}\text { Profile } \\
\text { No }\end{array}$ & Depth & EC & Texture & Slope & Drainage & $\mathrm{CaCO}_{3}$ & Gypsum & ${ }^{*} \mathrm{Ci} \%$ & Class \\
\hline \multirow[t]{30}{*}{ PI 111} & 1 & 1 & 0.85 & 0.6 & 1 & 1 & 1 & 0.96 & 49.0 & S3 \\
\hline & 2 & 1 & 0.98 & 0.6 & 1 & 1 & 1 & 1 & 58.8 & S2 \\
\hline & 3 & 1 & 0.88 & 0.6 & 1 & 1 & 1 & 1 & 52.8 & $\mathrm{~S} 2$ \\
\hline & 4 & 1 & 0.96 & 0.7 & 1 & 1 & 1 & 1 & 67.2 & $\mathrm{~S} 2$ \\
\hline & 5 & 1 & 0.88 & 0.7 & 1 & 1 & 1 & 1 & 61.6 & $\mathrm{~S} 2$ \\
\hline & 6 & 1 & 0.88 & 0.7 & 1 & 1 & 1 & 1 & 61.6 & S2 \\
\hline & 7 & 1 & 0.88 & 0.6 & 1 & 1 & 1 & 1 & 52.8 & $\mathrm{~S} 2$ \\
\hline & 8 & 1 & 0.96 & 0.7 & 1 & 1 & 1 & 1 & 67.2 & $\mathrm{~S} 2$ \\
\hline & 9 & 1 & 0.96 & 0.6 & 1 & 1 & 1 & 1 & 57.6 & S2 \\
\hline & 10 & 1 & 0.96 & 0.7 & 1 & 1 & 1 & 0.96 & 64.5 & S2 \\
\hline & 19 & 1 & 0.98 & 0.6 & 1 & 1 & 1 & 0.96 & 56.4 & $\mathrm{~S} 2$ \\
\hline & 20 & 1 & 0.96 & 0.6 & 1 & 1 & 1 & 0.96 & 55.3 & $\mathrm{~S} 2$ \\
\hline & 21 & 1 & 0.96 & 0.6 & 1 & 1 & 1 & 1 & 57.6 & S2 \\
\hline & 22 & 1 & 0.96 & 0.6 & 1 & 1 & 1 & 1 & 57.6 & S2 \\
\hline & 23 & 1 & 0.96 & 0.6 & 1 & 1 & 1 & 1 & 57.6 & S2 \\
\hline & 24 & 1 & 0.96 & 0.6 & 1 & 1 & 1 & 1 & 57.6 & $\mathrm{~S} 2$ \\
\hline & 25 & 1 & 0.9 & 0.6 & 1 & 1 & 1 & 1 & 54.0 & S2 \\
\hline & 26 & 1 & 0.9 & 0.6 & 1 & 1 & 1 & 1 & 54.0 & S2 \\
\hline & 27 & 1 & 0.98 & 0.7 & 1 & 1 & 1 & 1 & 68.6 & $\mathrm{~S} 2$ \\
\hline & 28 & 1 & 0.98 & 0.6 & 1 & 1 & 1 & 1 & 58.8 & $\mathrm{~S} 2$ \\
\hline & 29 & 1 & 0.85 & 0.7 & 1 & 1 & 1 & 1 & 59.5 & S2 \\
\hline & 30 & 1 & 0.85 & 0.8 & 1 & 1 & 1 & 1 & 68.0 & S2 \\
\hline & 31 & 1 & 0.9 & 0.75 & 1 & 1 & 1 & 1 & 67.5 & S2 \\
\hline & 32 & 1 & 0.9 & 0.75 & 1 & 1 & 1 & 1 & 67.5 & S2 \\
\hline & 33 & 1 & 0.9 & 0.6 & 1 & 1 & 1 & 1 & 54.0 & $\mathrm{~S} 2$ \\
\hline & 34 & 1 & 0.9 & 0.6 & 1 & 1 & 1 & 1 & 54.0 & $\mathrm{~S} 2$ \\
\hline & 35 & 1 & 0.96 & 0.7 & 1 & 1 & 1 & 1 & 67.2 & S2 \\
\hline & 36 & 1 & 0.9 & 0.6 & 1 & 1 & 1 & 1 & 54.0 & $\mathrm{~S} 2$ \\
\hline & 37 & 1 & 0.96 & 0.7 & 1 & 1 & 0.96 & 1 & 64.5 & $\mathrm{~S} 2$ \\
\hline & 38 & 1 & 0.96 & 0.6 & 1 & 1 & 0.96 & 1 & 55.3 & $\mathrm{~S} 2$ \\
\hline \multirow[t]{13}{*}{$\begin{array}{c}\mathrm{Hi} \\
112 \\
\end{array}$} & 11 & 0.6 & 0.8 & 0.6 & 0.9 & 0.96 & 1 & 1 & 25.0 & S3 \\
\hline & 12 & 0.6 & 0.8 & 0.6 & 0.9 & 0.96 & 1 & 1 & 25.0 & S3 \\
\hline & 13 & 0.85 & 0.8 & 0.6 & 0.9 & 0.96 & 1 & 1 & 35.3 & S3 \\
\hline & 14 & 0.6 & 0.75 & 0.6 & 0.9 & 0.96 & 1 & 1 & 23.3 & $\mathrm{~N} 1$ \\
\hline & 15 & 0.6 & 0.75 & 0.6 & 0.9 & 0.96 & 1 & 1 & 23.3 & N1 \\
\hline & 16 & 0.85 & $\begin{array}{l}0.8 \\
\end{array}$ & 0.6 & 0.9 & 0.96 & 1 & 1 & 35.3 & S3 \\
\hline & 17 & 0.85 & 0.8 & 0.6 & 0.9 & 0.96 & 1 & 1 & 35.3 & S3 \\
\hline & 18 & - & - & - & - & - & - & - & - & N2 \\
\hline & 39 & - & - & - & - & - & - & - & - & N2 \\
\hline & 40 & 0.75 & 0.8 & 0.6 & 0.9 & 0.96 & 1 & 1 & 31.1 & $\mathrm{~S} 3$ \\
\hline & 41 & & & & & & $=$ & $=$ & - & N2 \\
\hline & 42 & 0.6 & 0.8 & 0.6 & 0.9 & 0.96 & 1 & 1 & 25.0 & S3 \\
\hline & 43 & 0.6 & 0.8 & 0.6 & 0.9 & 0.96 & 1 & 1 & 25.0 & S3 \\
\hline $\begin{array}{c}\mathrm{Hi} \\
111 \\
\end{array}$ & & - & - & - & - & - & - & - & - & N2 \\
\hline $\begin{array}{c}\mathrm{Du} \\
111 \\
\end{array}$ & & - & - & - & - & - & - & - & - & N2 \\
\hline
\end{tabular}

${ }^{*} \mathrm{Ci}=$ Capability index 


\section{Land suitability for specific crops:}

Land suitability for five different crops, i.e. Wheat, Barley, Maize, Tomato and Olive was tested for the soils using Arc GIS 10.2 software. The results were imported to Arc GIS to display maps. Soil characteristics of the different mapping units were compared and matched with the crop requirements of each land use type, i.e. crop (FAO, 1976 b). The matching led to the current and potential suitability for each crop using the parametric approach and land index as mentioned by Sys et. al. (1993) (Table 7-8 and Figures 7-11).

\subsection{Current suitability}

The data in Table (8) and Figures (7, 9 and 11) show the current sutabitity classes for the selected studied crops. These data indicate that $67.4 \%$ is highly suitable $\left(\mathrm{S}_{1}\right)$ for olive. On the other hand, $67.4 \%$ is moderately marginally suitable $\left(\mathrm{S}_{2}\right)$ for wheat, barley, maize and tomato. The table shows that $26.16 \%\left(\mathrm{~S}_{3}\right)$ is only suitable for wheat, Barley and maize. Tomato is not suitable only for $\mathrm{N}_{1}(26.16 \%)$. The area of permenanty not suitable for all crops $\left(\mathrm{N}_{2}\right)$ is $6.44 \%$.

Table 8. Current suitability classes and areas \% for growing crops in the study area

\begin{tabular}{|c|c|c|c|c|c|}
\hline Suitability class* & Wheat & Barley & Maize & Tomato & Olive \\
\hline S1 & & & & & $67.4 \%$ \\
\hline S2 & $67.4 \%$ & $67.4 \%$ & $67.4 \%$ & $67.4 \%$ & \\
\hline S3 & $26.16 \%$ & $26.16 \%$ & $26.16 \%$ & & $26.16 \%$ \\
\hline N1 & & & & $26.16 \%$ & \\
\hline N2 & $6.44 \%$ & $6.44 \%$ & $6.44 \%$ & $6.44 \%$ & $6.44 \%$ \\
\hline $\begin{array}{r}* S_{1}=\text { Highly suitable, } \\
N_{1}=\text { Currently not suitable }\end{array}$ & $\mathrm{S}_{2}=$ Moderately suitable \\
\hline
\end{tabular}

4.2. Potential suitability

From the previous discussion, the main limiting factors were texture and salinity which can be improved using good management practices such as salt leaching, use of organic matter amendments, construction of a good drainage system and follow good agriculture practices for crops. These improvements will raise the potential suitability.

The results in Table (9) and Figures ( 8,10 and 11) show the area \% of the potential suitability classes. The data show that $93.56 \%$ of the area is moderately suitable $\left(\mathrm{S}_{2}\right)$ for wheat, barley and maize, while an area of about $6.44 \%$ is permanently not suitable $\left(\mathrm{N}_{2}\right)$ for all crops.

Table 9. Potential suitability classes and areas \% for growing crops in the study area

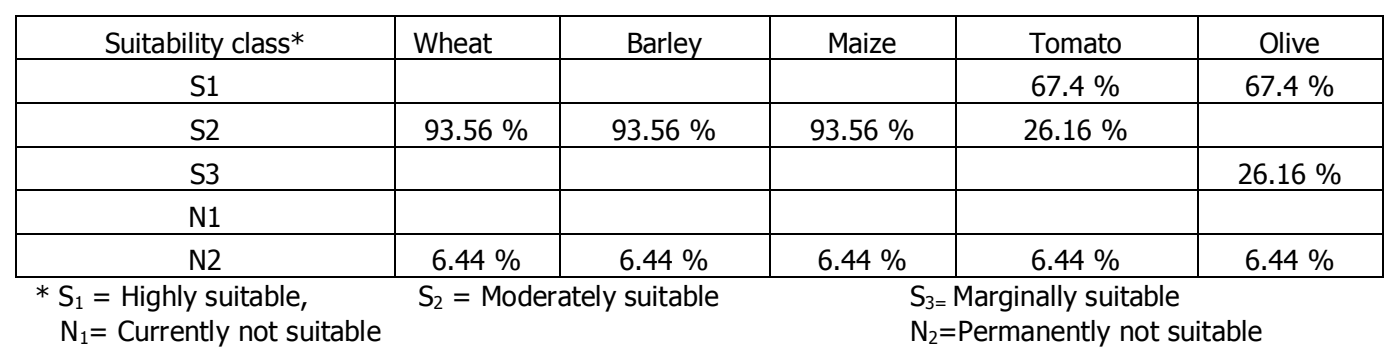




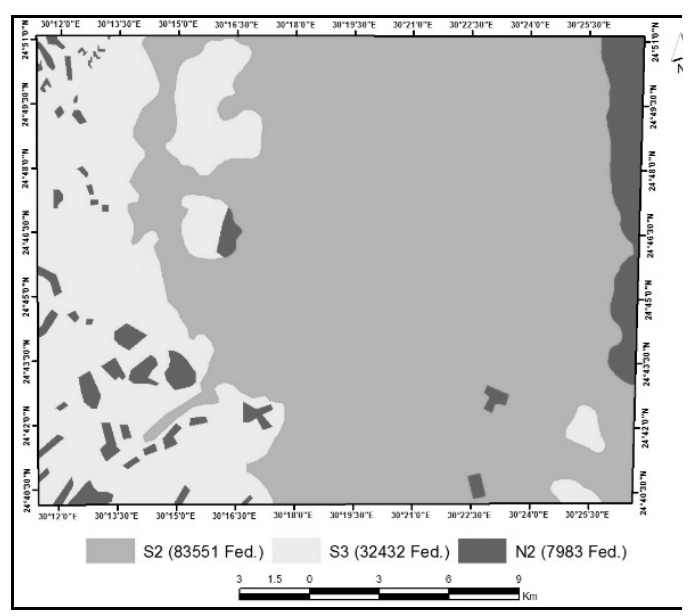

Figure 7. Current land suitability of wheat, barley and maize in the study area.

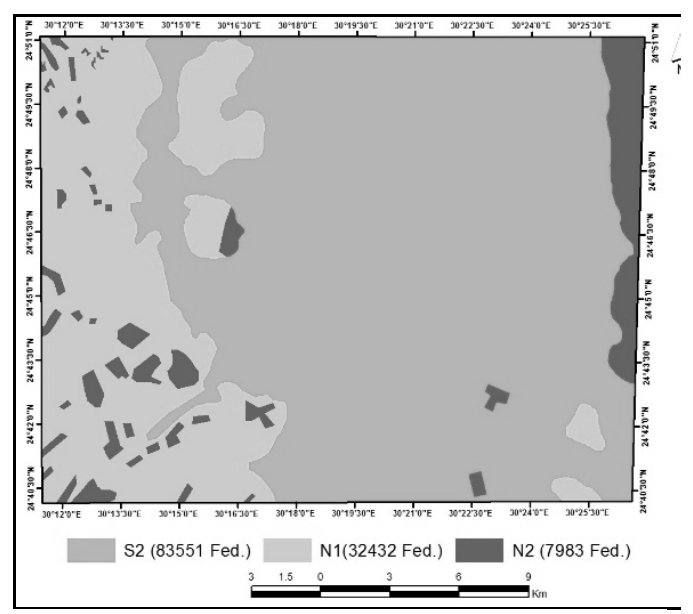

Figure 9. Current land suitability of tomato in the study area.

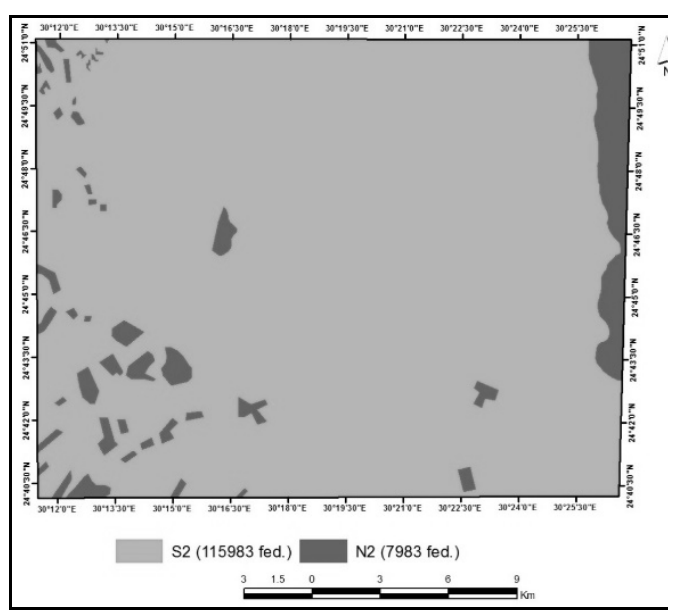

Figure 8. Potential land suitability of wheat, barley and maize in the study area.

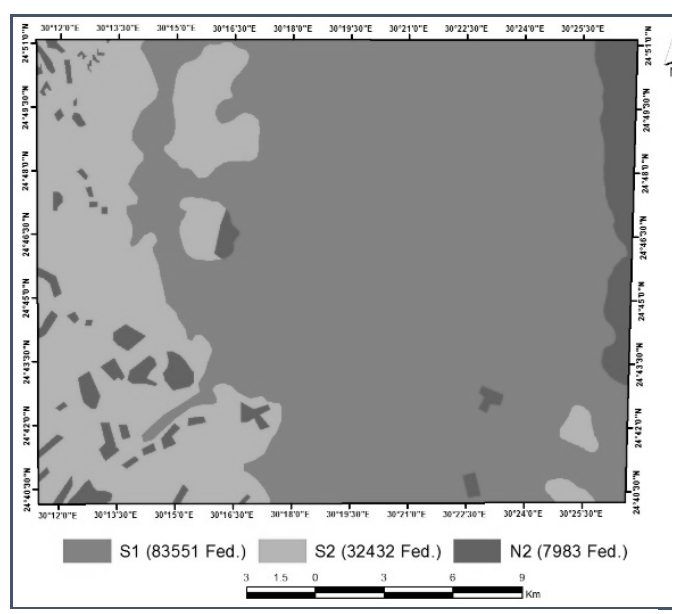

Figure 10. Potential land suitability of tomato in the study area.

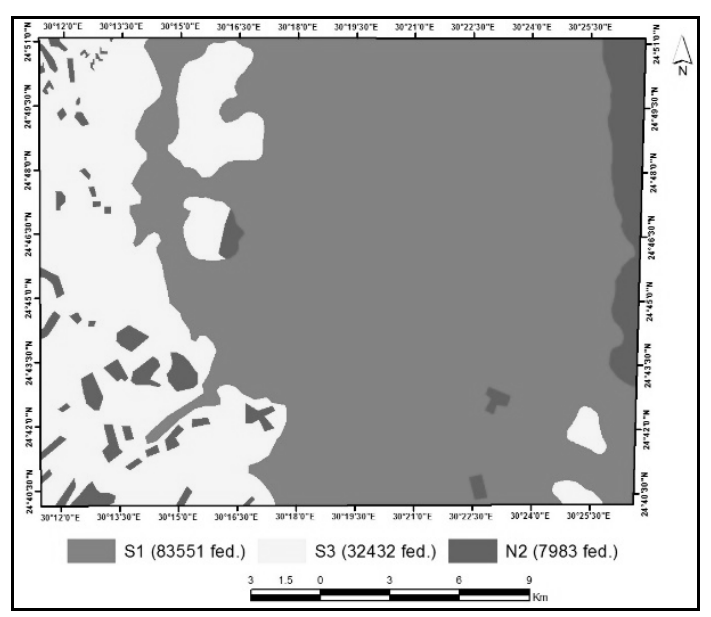

Figure 11. Current and potential land suitability of olive in the study area. 


\section{REFERENCES}

1. Black, C. A. 1982. Methods of Soil Analysis. Part 2, Chemical and Microbiological Properties. Agronomy series No. 9, ASA, SSSA, Madison, Washington, USA, 720 p.

2. Daels, L. 1986. Remote sensing fundamentals. Gent. State Univ., Gent., ITC. J., pp.1-19.

3. Darwish Kh. M., M. M. Wahba and F. Awad. 2006. Agricultural Soil Suitability of soils for Some Crops in Newly Reclaimed Areas of Egypt. Journal of Applied Sciences Research, 2, 1235-1243.

4. Dent, D. and A. Young. 1981. Soil Survey and Land Evaluation. George Allen and Unwin, London.

5. DeVries, M. E. 1985. Use of GIS to integrate remote sensing and other natural resources data 18th International Symposium on Remote Sensing of Environment, Arbor, Michigan, International J. Remote Sensing, 31:71-90.

6. EGSA (Egyptian Geological Survey Authority) 1988. "Egyptian General Petroleum Corporation: Geological Map of Egypt", Conoco Coral, printed in Germany by institute fur Angewandte Geodasie, Berlin, Technische Fachhochschule Berlin, 1988, Scale 1:500000.

7. Elachi C. and J. V. Zyl 2006. Introduction to the Physics and Techniques of Remote Sensing. 2nd Edition. John Wiley \& Sons Inc.

8. FAO 1976 a. Report on the Agro-ecological zones project. World Soil Resources Report 48. FAO, Rome

9. FAO 1976 b. A framework for land evaluation. FAO Soil Bulletin, 32. Rome, Italy.

10. FAO 1985. Guidelines: Land Evaluation for irrigated Agriculture. FAO Soils Bulletin No. 55, Rome, Italy.

11. FAO 2006. "Guidelines for soil description "fourth edition, FAO, Rome, ISBN 92-5105521-10.

12. Jackson, M. L. 1976. Soil Chemical Analysis. Prentice Hall of India, New Delhi, India, $930 \mathrm{p}$.

13. Meteorological Authority 2014. The Normals for Egypt up to 2014. Ministry of Civil Aviation, Cairo, Egypt. Meteorological Res. Bull., 32:55-65.

14. Moussa, M. A. 1991. Land suitability evaluation of El Saff area eastern desert Egypt for agriculture utilization. Ph.D. Thesis, Fac. of Agric. Zagazig Univ. Benha Branch, Egypt.

15. MSA, 1986. Topographic map scale 1: 100,000", edition by Military Survey Authority 1986. based on soil survey 1970. 
16. O'Geen A. T. and S. B. Southard 2005. A revised Storie index model in NASIS. Soil Survey 46 (3):98-109.

17. Piper, C. S. 1950. Soil and Plant Analysis. Univ. of Adelaide press, Adelaide, Australia, $368 \mathrm{p}$.

18. Storie, R. 1978. Storie index rating. Oakland: University of California Division of Agricultural Sciences Special Publication 3203.

19. Sys, C., E. Van Ranst and J. Debavey. 1991. Land Evaluation. Part I and S2, Ghent Univ., Ghent Belgium.

20. Sys, C., E. Van Ranst, J. Debavey and F. Beeranert. 1993. Land_ Evaluation. Part III Crops Requirements, Ghent Univ., Ghent Belgium., The Netherlands, 190 p.

21. Sys, C. and Verheye, W. (1978). An attempt to the evaluation of physical land characteristics for irrigation according to the FAO Framework for land evaluation. Ghent, Belgium., The Netherlands, ITC. J., pp. 66-78.

22. UNDP-UNESCO 2005. Geomorphological maps of Western Desert and Sinai.

23. USDA 2010. Keys to Soil Taxonomy. U.S.D.A., Soil Cons Serv. Washington.

24. Zhongxin C., Sen Li, R. Jianqiang, P. Gong and M. Zhang 2004. Monitoring and Management of Agriculture with Remote Sensing. In: Liang S. (Ed.), Advances in Land Remote Sensing. Springer, 397-421.

25. Zinck, J. A. 1988. Geomorphology and Soils. Internal Publ., ITC., Enschede, The Netherlands. 


\section{تقييم التربة باستخدام نموذجى Sys - Storie Index في بعض مناطق}

شمال غرب واحات باريس -مصر

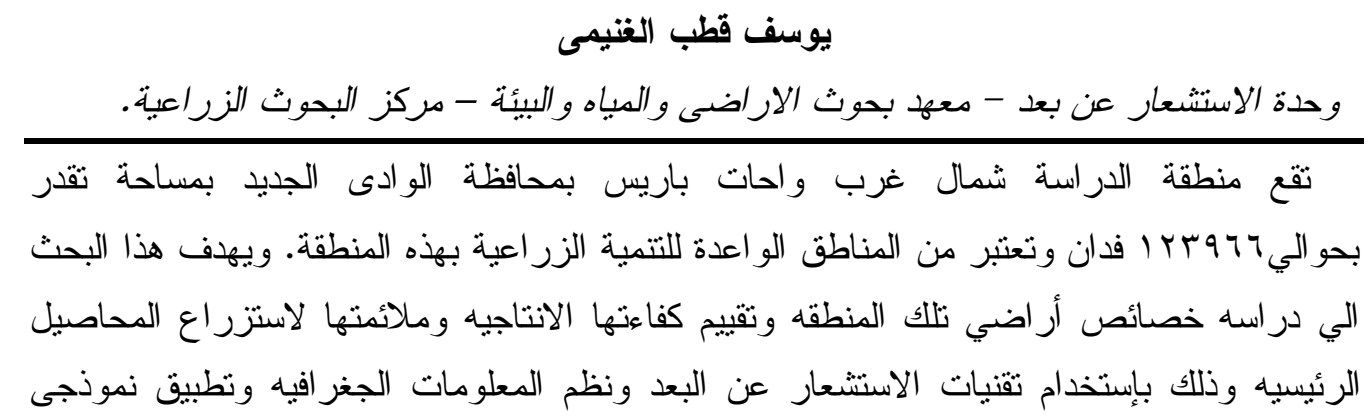

Storie Index- Sys تقييم الار اضي الجافه

ولهذا الغرض نم إختيار وحفر rع قطاعا أرضيا ممثلا لار اضي المنطقه ، . r حفرة صغيرة ،

ولقد وصفت هذه القطاعات مورفولوجيا وجمعت منها عينات تمثل الاختلافات الر أسيه لها للتحليلات

$$
\text { المعمليه. }
$$

ولقد تم عمل خريطة فيزيوجر افية باستخدام التفسير المرئي لصورة القمر الصناعي لاندسات 1 ردات

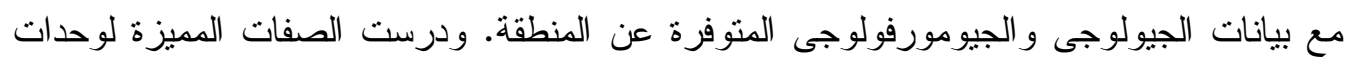
خريطة التربة المنتجة وتم التعرف على الوحدات التصنيفية السائدة بها.

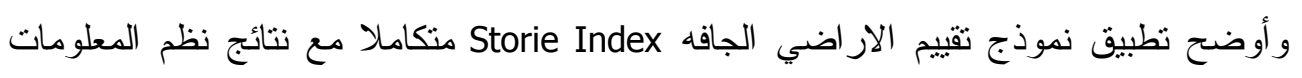

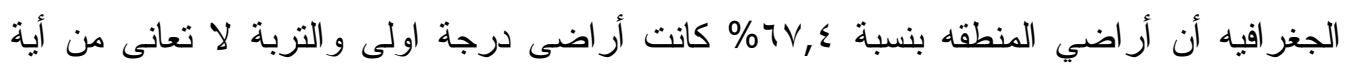

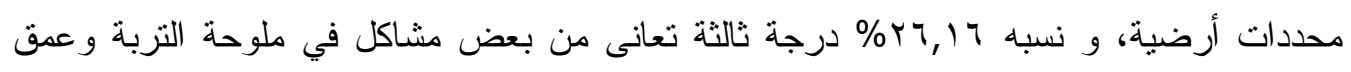

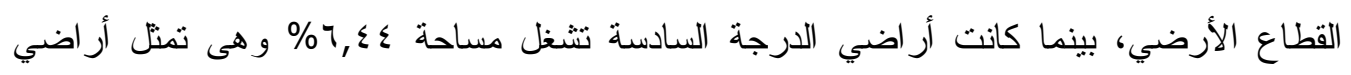

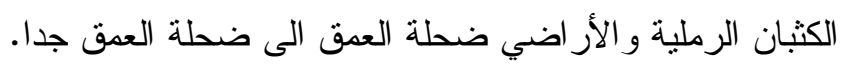

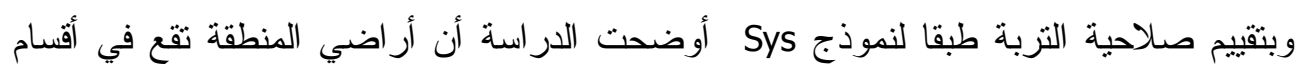

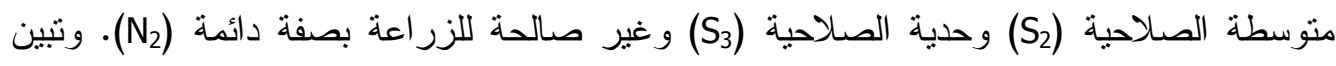
النتائج أن حو الي گ, TV \% من اجمالي منطقة الدراسة هي أراضي منوسطة الصلاحية (S2) و وأن

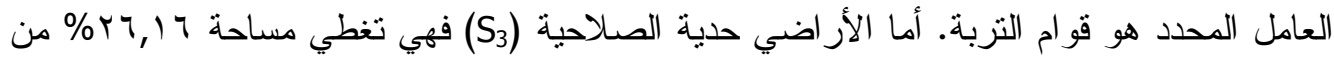

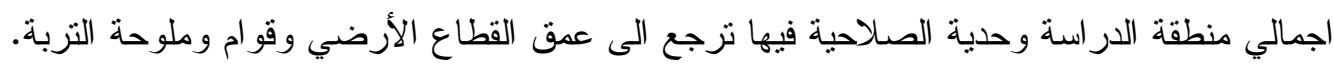

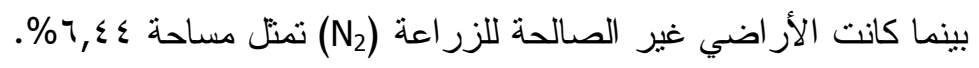

وقد تم اختيار خمسة محاصيل لتقييم درجة صلاحيتها للزر اعة طبقا لطريقة Sys وهي و القمح

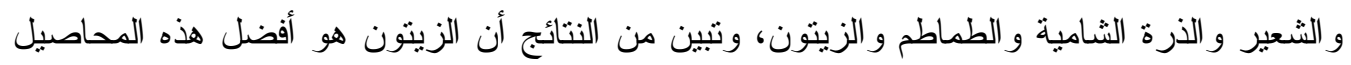
حيث تجود زر اعته بدرجة أعلى من باقي المحاصيل. 